\title{
GPS Calibrated Ad-Hoc Localization for Geosocial Networking
}

\author{
Dexter H. Hu, Cho-Li Wang, and Yinfeng Wang \\ Department of Computer Science, \\ The University of Hong Kong, \\ Pokfulam Road, Hong Kong \\ \{hyhu, clwang, yf wang\}@cs.hku.hk
}

\begin{abstract}
Cost-effective localization for large-scale Geosocial networking service is a challenging issue in urban environment. This paper studies an ad-hoc localization technique which takes advantages of short-range interchanged location information for calibrating the location of mobile users carrying non-GPS mobile phones. We demonstrate by simulation that a small percentage of GPS-enabled mobile phones can greatly enable the localization of other non-GPS pedestrians in the urban environment. Based on the proposed localization technique, we implement a location-aware social networking tool called Mobile Twitter, similar to the microblogging service of Twitter, for fast propagation of social events happening in surroundings. Evaluation shows the our localization algorithm can achieve better accuracy of the location estimation and wider coverage as compared with the Amorphous algorithm and the Monte Carlo Localization (MCL) method. Moreover, we show that the Mobile Twitter implemented on an Android mobile phone is power-efficient in real-life usage scenarios.
\end{abstract}

\section{Introduction}

In recent years, Geosocial networking 4] has emerged as a new type of social networking application, by which various geographic services, such as nearby activity recommendation, event planning of friends in the same area, are provided to enable meaningful social dynamics in the real world. Mobile social networking has long be existing for years. However without location awareness, mobile social networking will be merely a simple extension to access social network websites [1,2 from a mobile device. In fact there are many interesting geosocial events that are first discovered by ordinary mobile phone users, which are better off to be quickly informed to community, similarly to the social networking and microblogging service of Twitter [3], which gains popularity because it can deliver the freshest information quickly online. For example, Google Buzz 11 has quickly become one of most popular location-based service on the Internet, which allows users to see messages from nearby users. Therefore, localization is core building

${ }^{1}$ http://www.google.com/buzz/help/privacy.html

Z. Yu et al. (Eds.): UIC 2010, LNCS 6406, pp. 52 66, 2010.

(C) Springer-Verlag Berlin Heidelberg 2010 
block of these Location-Based Services (LBS). Without location information and the willingness of location information sharing, mobile social networking is not possible.

The most well-known and matured localization technique nowadays is the Global Positioning System (GPS) 20 Due to the advancement of hardware manufacturing, more and more affordable mobile phones (e.g., iPhone 3G, Nexus One) come with a built-in GPS unit, which enables real-time tracking of user location. Many novel location-based services become a reality. According to ABI research 5, it is estimated that the number of mobile phone subscribers with GPS equipped devices will grow to $9 \%$ by the year 2011. So we could expect that only a small percentage of mobile phones have GPS functions in the near future, while the rest majority are without. This is one of the obstacles that hurdle the practicality of modern location-based services in people's daily life. One intuitive solution to this urban localization problem is allowing people with GPS to collaboratively help calibrate the localization of those without, by using ad hoc connectivity information in an urban district. Fortunately, ad hoc communication between mobile users can be done by Personal Area Network (PAN) wireless communication (e.g., ZigBee, Bluetooth).

In this paper, we first proposed a new GPS-calibrated ad-hoc localization algorithm named MobiAmorph, which takes advantages of short range interchanged information among mobile phones, so that many non-GPS mobile phone users will now be able to localize themselves based on information from nearby users. Second, we have done intensive experiments to show that MobiAmorph are superior than two typical distributed locations algorithms in urban environment. Third, we developed the Mobile Twitter application that are practical for real life usage and can encourage ad-hoc information sharing, so that mobile social applications will be more meaningful and location-aware (e.g., twitting social news with location information attached). With the help of GPS-enabled mobile users, localization is possible without the deployment of large infrastructure, hence, will boost its adoption. Also under certain mobility model, accurate GPS information can quickly propagate to non-GPS users, which is suitable for pedestrians in typical urban environment.

The rest of this paper is organized as follows. We review related work of localization approaches in Section 2. We explain the design of Mobile Twitter application in Section 3. Our proposed localization algorithm is explained in Section 4. We report the evaluation of algorithms under different experiment settings in Section 5 . Section 7 concludes the paper with future work.

\section{Related Work}

Localization in general has long been a fundamental problem in Mobile Ad Hoc Networks (MANET) [6] and Wireless Sensor Networks (WSN) 7], on which many routing principles and applications rely. For instance, geographic routing in WSN needs location information as a priori to route message without

\footnotetext{
2 http://en.wikipedia.org/wiki/GPS
} 
knowledge of network topology. Many wireless applications that are related to monitoring and control, require accurate location information, such as wildlife habitat tracking, intrusion detection, disaster control, etc.

Existing distributed localization techniques can be divided into range-free and range-based approaches. Range-based approach makes use of additional hardware to obtain information like angle of arrival [8] and received signal strength 9, which may be affected by fading and introduce distance estimation error. This approach is generally expensive because of specially required hardware. Range-free localization approach, on the other hand, is a cost effective alternative, which uses location dissemination of seed with moderate to high density, and packet relaying of normal nodes, so that they can receive enough information to do self-localization. Our proposed algorithm falls in this category. MCL 10 and Amorphous [11 are two typical range-free localization algorithms, which are studied and compared in this paper.

The Monte Carlo Localization (MCL) technique represents the posterior distribution of a node's possible locations using a set of weighted samples. MCL internally maintains a set of $N$ location samples in the deployment area, which are initially selected randomly from all possible locations. In the Prediction phase, it tries to compute a new possible location set based on the location set at the previous time step using maximum velocity. At the same time, MCL will get new information from seed nodes. MCL exchanges packets of seed with a maximum hop count value of two. Filtering step will eliminate those impossible predicted locations that are inconsistent with seed information. Re-sampling is performed to maintain enough $N$ location samples after Filtering. Generally MCL is suitable for situation of nodes with high mobility, because a node cannot locate itself if there are no seeds within two hop distance and the algorithm can be very inaccurate in the beginning when it does not have enough samples to guess an accurate position.

Amorphous algorithm and its similar variant DV-HOP 12 are range-free localization algorithms based on hop-counting technique, which is similar to distance vector routing. Each seed broadcasts its location to neighbors and other nodes try to estimate their distance to seeds. The nodes will store the coordinates of enough number of seeds, which are propagated throughout the network and maintain the current minimum hop count to those seeds. Nodes calculate their position based on the received seed location information packets and corresponding hop count value, which is called the multilateration procedure. Amorphous algorithm has higher network coverage, (i.e. it can localize more nodes) and low requirement of the number of seed nodes. However, it can induce very high network traffic and too static for mobile situations in urban environment.

\section{Mobile Twitter: Architecture Design}

This section explains the Mobile Twitter application that uses "Ad Hoc Location Tracking" technique. Mobile Twitter is a geosocial networking software which motivates proactive social event propagation, and increase user incentive 
for location information sharing. It can be applied to many situations such as advertising, taxi call, ad hoc voting, and disaster/rescue systems. We have implemented it on Android 3 mobile platform in a street situation, where one user can quickly propagate a nearby news event attached with estimated location, allowing emergent or interesting social event to be shared quickly with others. It enables people with GPS-enabled phone to send instant messages with their GPS information to others through Bluetooth. Then the receiver can receive the GPS information and forward sender's message immediately. Mobile Twitter can calculate the current location and forward that message to others who are nearby and have switched on the same application. For the Non-GPS enabled phones, they can also create and send their new message to public through Bluetooth once their current location is calculated.

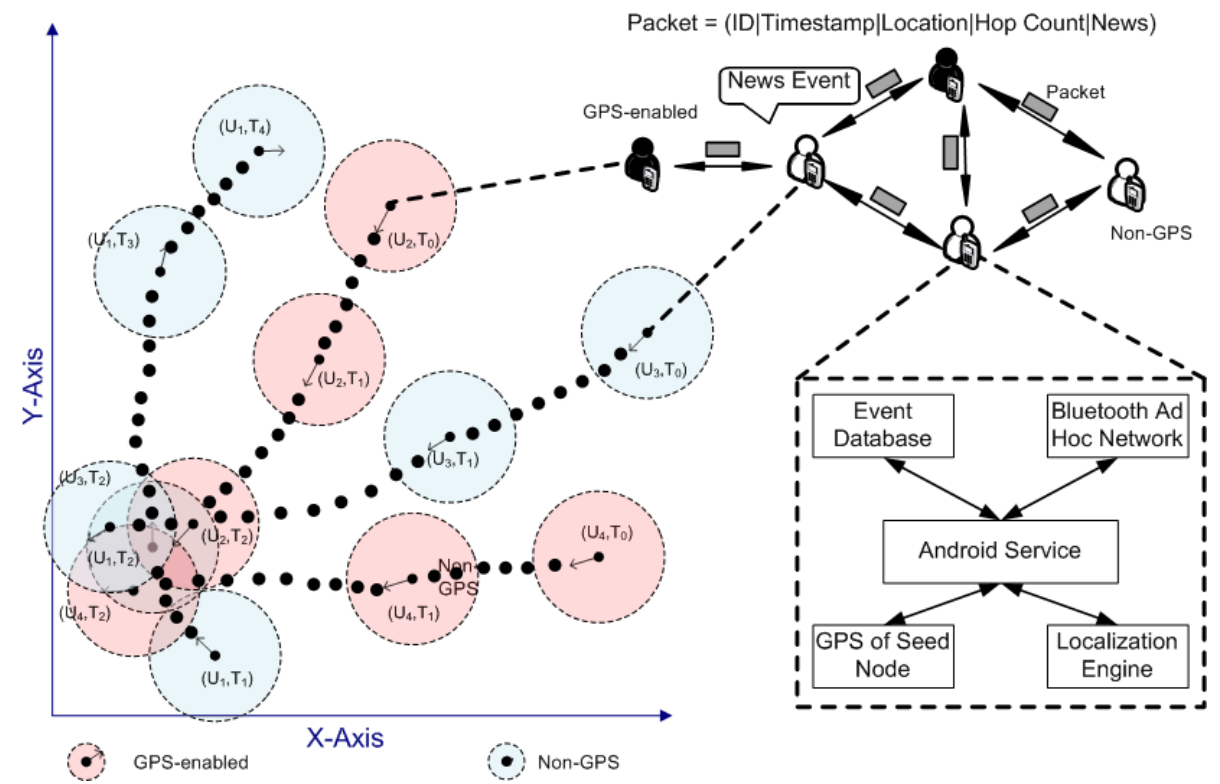

Fig. 1. Mobile Twitter Usage Scenario and Components

Figure 3 shows a typical usage scenario and different components within Mobile Twitter. The black mobile users have GPS enabled phones, while the white mobile user's phone does not have GPS function. All white mobile phones running Mobile Twitter application will be able to localize themselves upon receiving enough nearby location information packets, with the MobiAmorph algorithm explained in the next section. There is an Android Service process that accesses the Localization Engine of non-GPS nodes or directly get location information of GPS-enabled nodes. The Bluetooth Ad Hoc Network component will broadcast out user's (estimated) location or forward location packet received from nearby

3 http://developer.android.com/index.html 
nodes. It also broadcasts the application content (e.g. emergent news) stored in the Event Database to form a mobile social network. Mobile Twitter can visualize the news event on Google Maps with its estimated location attached by the first observer.

\section{GPS Calibrated Ad Hoc Localization}

We proposed a new GPS-calibrated localization algorithm, named MobiAmorph, based on the Amorphous algorithm [11, as shown in Algorithm 1. Table 1] summarizes common notations used in later discussion.

Table 1. Common Notations

\begin{tabular}{|l|l|}
\hline Terminologies & Explanations \\
\hline Node & user with Non-GPS mobile phone and willing to relay packets \\
\hline Seed & $\begin{array}{l}\text { user with GPS-enabled mobile phone and willing to share } \\
\text { location information and relay packets }\end{array}$ \\
\hline Hop Count & $\begin{array}{l}\text { the number of hop unit that a packet need to travel between nodes } \\
\text { there is a maximum hop count limit } H_{m a x}\end{array}$ \\
\hline Accuracy & $\begin{array}{l}\text { A.k.a Average Position Error (APE), the average of all node's } \\
\text { (non-seed) location estimation errors in meters }\end{array}$ \\
\hline Seed Ratio & Percentage of seeds among all nodes including seeds and nodes. \\
\hline Packet Interval & $\begin{array}{l}\text { Frequency at which each node broadcasts/forwards packet once } \\
\text { every interval of } t \text { seconds }\end{array}$ \\
\hline Coverage & $\begin{array}{l}\text { Percentage of nodes within non-seed nodes with position } \\
\text { estimation at the end of a packet interval } t \text { (i.e., localizable). }\end{array}$ \\
\hline Density & Number of nodes within the $10000 m^{2}$ area. \\
\hline Node Speed & The speed of a node or seed in $m / s$, \\
\hline Radio Range & $\begin{array}{l}\text { The radius of a node's communication circle by ZigBee or } \\
\text { Bluetooth (i.e., assuming no degree of irregularity) }\end{array}$ \\
\hline
\end{tabular}

The MobiAmorph algorithm addresses several weaknesses of Amorphous by relaxing trilateration criteria with additional historical data of past location estimates.

- Relaxed Trilateration: multilateration of Amorphous needs at least three reference points. Location estimating with overlapping circles can still have a decent estimation even there are only two reference points available. This will increase the chance of a node to calculate its position (i.e. increased coverage).

- Historical Data: it uses the last estimated location, to increase accuracy and coverage. Originally we cannot start calculation until there are at least three reference information received. Now, with last estimated location as a reference point, one can start estimating with only two reference information received. Together with relaxed trilateration, only one reference information 


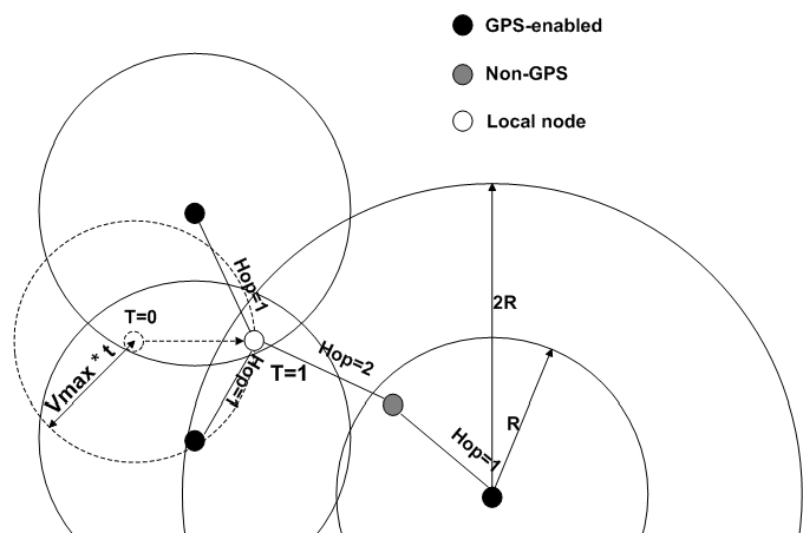

Fig. 2. Localization Considering Historical Data and Moving Velocity

is necessary to start estimation. For example, as shown in Figure 2, where $T$ is the elapsed time from previous localized point and $V_{\max }$ is the maximum possible node speed. Originally, the estimated distance between seed and current location (radius $r$ ) equals to $h * \mathrm{R}$ (radio range). Now we have another reference circle with radius $r=T * V_{\max }$. This can overcome Amorphous's weakness of lack of mobility, as MobiAmorph now have a better estimation of current position base on historic data even when the user moves to a sparse area.

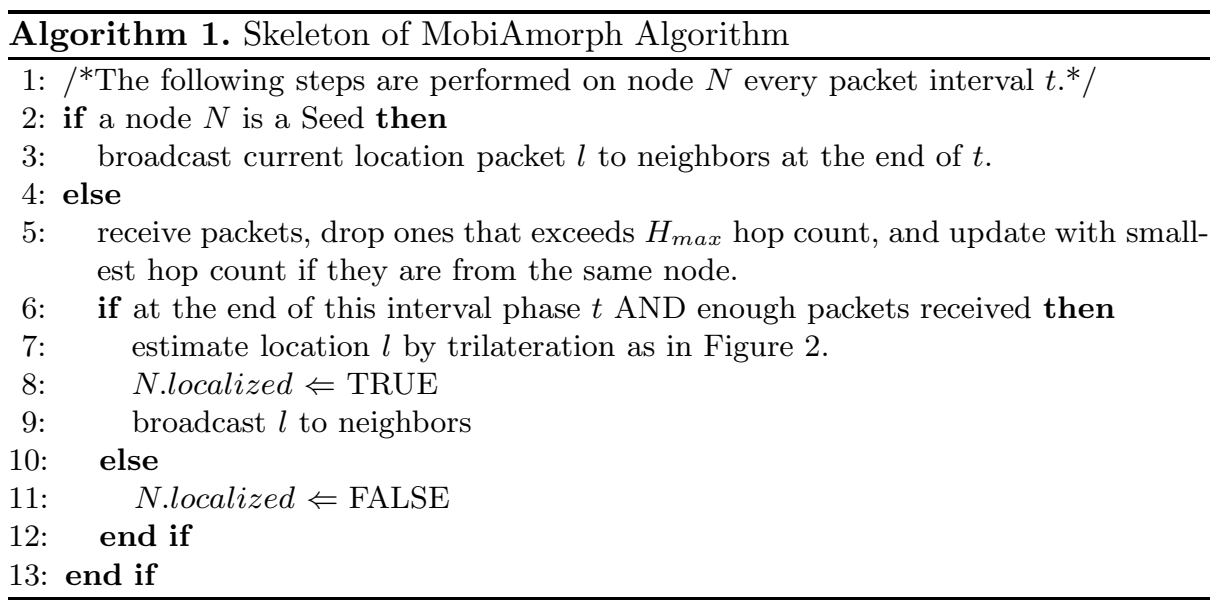

MobiAmorph will accumulate packet during a packet interval (Line 5), and start trilateration if enough packet received at the end of that interval (Line 6). Line 8 and 11 record if a node is localizable in that interval and will be used to 
calculate coverage. It has a maximum hop count $H_{\max }$ of two, which can help avoid error accumulation and reduce the number of flooded packets.

\section{Performance Evaluation and Analysis}

We used a network simulator called MobiREAL 13 that can simulate the ad hoc network with realistic mobility of humans with mobile devices in the city. It can change their behavior depending on a given application context. We implemented the MobiAmorph algorithm in MobiREAL and report the detailed performance evaluation in this section. The goals of the evaluation are multi-fold:

1. compare coverage and accuracy of MobiAmorph with other well known ones under both street scenario and open area environments.

2. analyze MobiAmorph's coverage and accuracy affected by various factors, in order to get the recommended configuration for real life deployment.

3. evaluate the feasibility of the algorithm within the Mobile Twitter, considering power consumed by computation and communication on real devices.

\subsection{Performance Comparison with Other Existing Algorithms}

We evaluated three algorithms (namely, Amorphous, MCL, MobiAmorph) under both street area and open area scenarios. The street scenario is a $500 \mathrm{~m} \times 500 \mathrm{~m}$ city area with buildings from TRACKIE [14] as in Figure 3(a), and the open area scenario is a $100 \mathrm{~m} \times 100 \mathrm{~m}$ square as in Figure 3(b). The gray shapes are buildings, green dots are non-seed users (nodes), and red dots are GPS-enabled (seeds) users. The semitransparent area of each dot is the radio range, which is a perfect circle right now. The radio range of Bluetooth is around $10 \mathrm{~m}$. We set the $V_{\max }$ to be $5 \mathrm{~m} / \mathrm{s}$, and the $H_{\max }$ to be 2 . All nodes move in the street randomly and independently. We focus on tuning parameters of seed ratio, packet interval and node speed. The bold value in Table 2 is set as fixed parameter when testing other parameters.

Table 2. Parameter Settings on Street Scenario

\begin{tabular}{|l|l|}
\hline Parameter & Value \\
\hline Node Speed $(\mathrm{m} / \mathrm{s})$ & $\mathbf{1 . 5}, 3,5$ \\
\hline Radio Range $(\mathrm{m})$ & $\mathbf{1 0}$ \\
\hline Seed Ratio & $0.2, \mathbf{0 . 3}, 0.4,0.5$ \\
\hline Packet Interval & $\mathbf{5 , 1 5 , 3 0 , 6 0 , 9 0}$ \\
\hline Density & $\mathbf{3 0}$ \\
\hline
\end{tabular}

Figure 4(a) and 4(b) illustrate the impact of packet interval to three algorithms under street scenario and open area environment respectively. MobiAmorph performs the best among the three. When the packet interval is between $5 s-90 s$, Amorphous has the APE of $24 m-90 m$, MCL has $22 m-89 m$, while 
MobiAmorph has $7 m-48 m$ for street scenario. Similar results are also shown for open area environment. Higher packet interval will increase APE rapidly for all algorithms. Since larger transmission intervals may result in less information about neighbors. For Amorphous, with a longer packet interval, seed information packets cannot be exchanged frequently, nodes cannot receive useful information indirectly. For MCL, it is difficult for nodes to exchange the position information, as it randomly picked a sample location for position estimation and then negatively influenced the next step of position estimation.

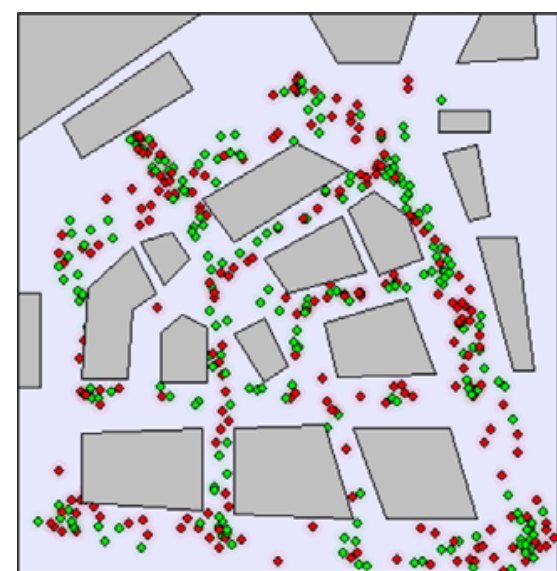

(a) Visualization of a $500 \mathrm{~m} \times 500 \mathrm{~m}$ Street Area

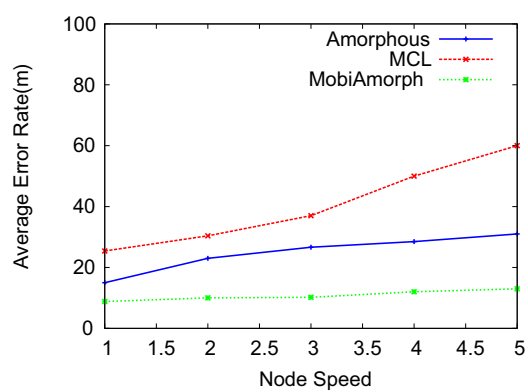

(c) APE vs. Node Speed in Street Scenario

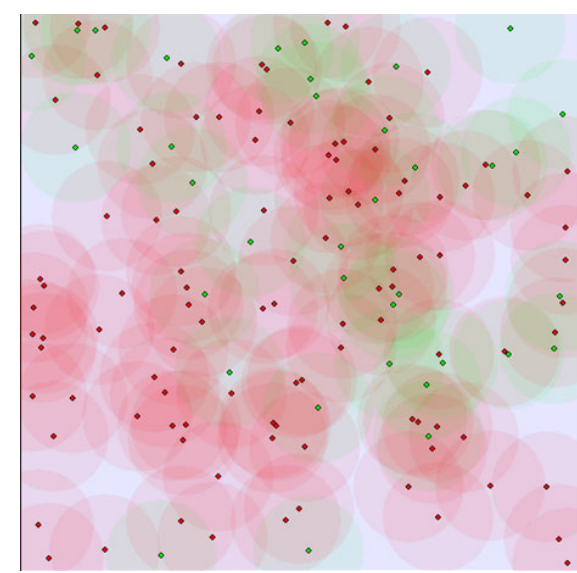

(b) Visualization of a $100 m \times 100 m$ Open Area

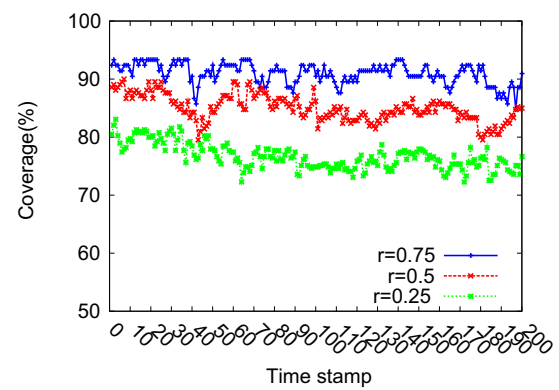

(d) Coverage vs. Seed Ratio over Time in Open Area

Fig. 3.

Figure 3(d) records the MobiAmorph's converge under different seed ratios in the open area environment. It shows that the higher the seed ratio, the higher the node coverage will consistently be over the time span of 200 seconds. 


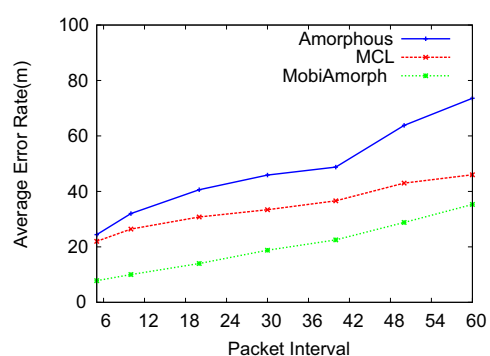

(a) APE vs. Packet Interval (Street)

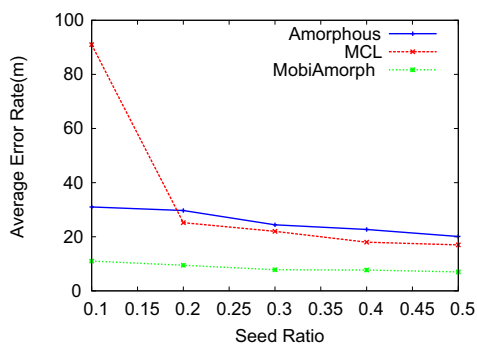

(c) APE vs. Seed Ratio (Street)

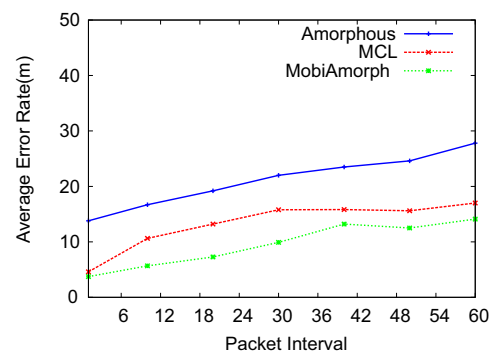

(b) APE vs. Packet Interval (Open Area)

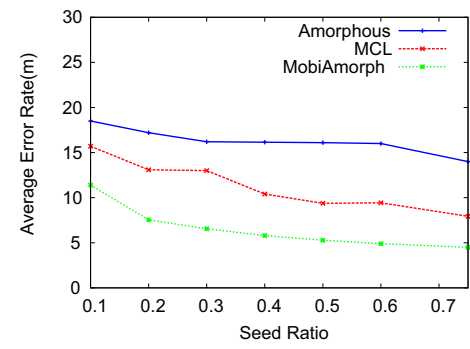

(d) APEvs.Seed Ratio(Open Area)

Fig. 4. Effect of Packet Interval and Seed Ratio in Street Scenario and Open Area

Figure 4(c) and 4(d) illustrate the effects of the seed ratio in street scenario and open area environment respectively. We can see MCL is affected much more than others, because its nodes need to encounter enough mobile landmarks (e.g. $N=15)$ to localize themselves. Amorphous needs to deploy more seeds to improve the accuracy because it needs stable ad-hoc networks where neighbors propagate seed position information. But it still has a relatively high error than MobiAmorph. If the seed ratio is very low (e.g., 0.2), nodes forward the packet received, after propagating certain hops, there will be large accumulated error. Moreover the calculation is based on the hop count information directly or indirectly received from the neighbor nodes, it prioritizes the seed information in the order of hop count.

Figure 3(c) illustrates the effects of node speed in street scenario. All algorithms have their APE increased with higher speed. MobiAmorph maintains low position error even when the node speed is high. Higher speed creates difficulties for receiving packets from neighbors. For MCL, APE is substantially increased from $22 \mathrm{~m}$ at $1.5 \mathrm{~m} / \mathrm{s}$ to $60 \mathrm{~m}$ at $5 \mathrm{~m} / \mathrm{s}$. This is because the uncertainty in the prediction phase becomes larger as node speed increases, which leads to wide range of position sample set and higher error. This is a side-effect of bad previous position sample set to current estimated position. Amorphous produces mild increase of average position error compared to MCL. Its error increases from $24.4 \mathrm{~m}$ at $1.5 \mathrm{~m} / \mathrm{s}$ to $31 \mathrm{~m}$ at $5 \mathrm{~m} / \mathrm{s}$. Amorphous is a localization technique that does not exploit historical information, and thus has less inaccurate influence. 
All in all, we can see that our MobiAmorph algorithm achieves higher accuracy than the other two. For MCL, it cannot get many seed information because of the two maximum hop count limit; while our algorithm has a higher chance of getting more reference information. In addition, our algorithm makes use of previous estimate location as one of the reference point. For normal pedestrian model, it is obvious that will help reduce the possible location of the local node and hence increase the accuracy of the estimated location.

\subsection{MobiAmorph Evaluation on Accuracy and Coverage}

This section reports the simulation result of MobiAmorph algorithm in terms of Accuracy (APE) and coverage affected by node density and speed in the street scenario. Because we want to find the optimal algorithm configuration that fits a typical real life urban environment, where there are buildings and streets involved. Unless with explicit notice, the bold value in Table 3 will be fixed when evaluating other parameters in a figure.

Table 3. Parameters Settings in Street Scenario for MobiAmorph

\begin{tabular}{|l|l|}
\hline Parameter & Value \\
\hline Packet Interval $(\mathrm{s})$ & $5, \mathbf{1 5}, 30,60,90$ \\
\hline Seed Ratio & $0.2, \mathbf{0 . 3}, 0.4,0.5$ \\
\hline Speed $(\mathrm{m} / \mathrm{s})$ & $\mathbf{1 . 5}, 3,5$ \\
\hline Node Density (per $\left.10000 \mathrm{~m}^{2}\right)$ & $10,20, \mathbf{3 0}, 40$ \\
\hline Radio Range $(\mathrm{m})$ & $\mathbf{1 0}$ \\
\hline
\end{tabular}

Figure 5(a) shows the effect to accuracy by varying packet interval under different seed ratio. The algorithm reduces APE to $11 \mathrm{~m}, 7.9 \mathrm{~m}, 7 \mathrm{~m}$ with packet interval at $15 \mathrm{~s}$. So to have a reasonable average position error within $20 \mathrm{~m}$, the packet interval should be less than 30 seconds. Figure $5(\mathrm{~b})$ shows the effect to accuracy by node speed under various node densities. We can see that the higher the density, the smaller the APE. However, a higher speed of node will increase the APE.

Figure 5(c) illustrates the effects on coverage by density in area of $10000 \mathrm{~m}^{2}$ under different seed ratio. The coverage is sensitive to node speed, because position calculation is related to packets exchange under suitable moving speed. Higher density also leads to higher chance of interchanging location information. At the same node speed, the higher node density, the higher coverage level. With speed at $1.5,3,5 \mathrm{~m} / \mathrm{s}$ respectively, MobiAmorph increases coverage from $5 \%$, $3 \%, 1 \%$ at low density of 10 to $54 \%, 27 \%, 10 \%$ at a higher density of 40 . To achieve reasonable coverage of $40 \%$, node speed $1.5 \mathrm{~m} / \mathrm{s}$ and density of more than 30 nodes in an area of $10000 \mathrm{~m}^{2}$ are recommended.

Figure $5(\mathrm{~d})$ illustrates the effects on coverage by packet interval under different seed ratios. Coverage is the percentage of localized non-seed nodes. MobiAmorph has high coverage when seed ratio is high, and packet interval is short, because 

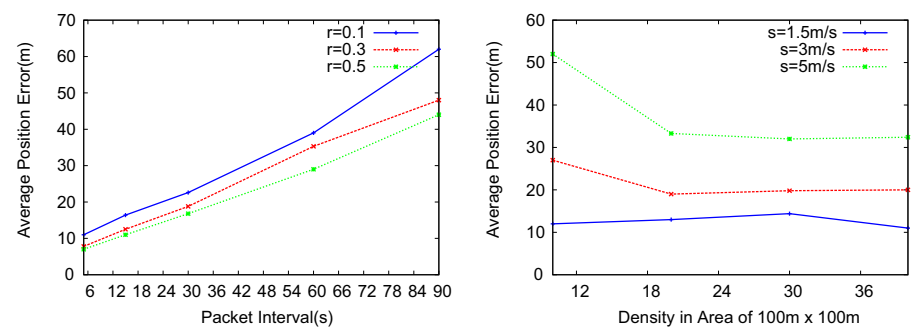

(a) APE vs. Packet Interval and (b) APE vs. Node Density and Seed Ratio

Seed Ratio
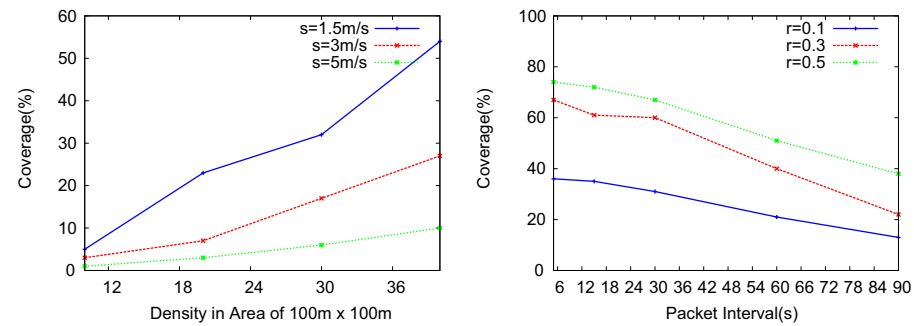

(c) Coverage vs. Node Density (d) Coverage vs. Packet Interval and Seed Ratio

and Seed Ratio

Fig. 5. MobiAmorph Algorithm Performance on Street Scenario

this situation provides higher opportunity for information exchange. With seed ratio at $0.1,0.3,0.5$ respectively, MobiAmorph decreased coverage from $35 \%$, $61 \%, 72 \%$ at packet interval $15 s$ to $13 \%, 22 \%, 38 \%$ at packet interval of $90 \mathrm{~s}$. To have a reasonable coverage of at least $40 \%$, the packet interval should be less than $60 \mathrm{~s}$, and the seed ratio should be at least $30 \%$.

\subsection{Traffic Overhead}

To be fair, the communication traffic overhead is measured as the average number of message a node needs to send in each localization step. Although the actual size of traffic packets can vary slightly due to encoding and encryption. The Amorphous algorithm needs seeds to flood their information through out the network, and each node needs to relay and broadcast a specified number of seed location (e.g., 32). Both the MCL and MobiAmorph can share a node's location to their neighbors, which are relatively much less than Amorphous.

\subsection{Mobile Twitter Deployment Evaluation}

Basically we conducted two experiments to evaluate the Mobile Twitter on real phone. We are interested in power consumption by sending location packets over Bluetooth, and also application's longevity over time by monitoring CPU and memory consumption when running Mobile Twitter on an Android phone. 


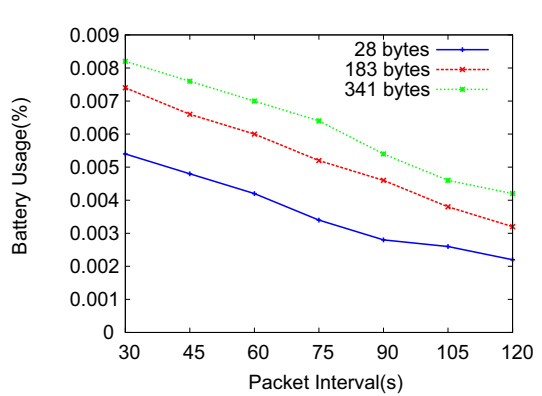

(a) Bluetooth Power Consumption

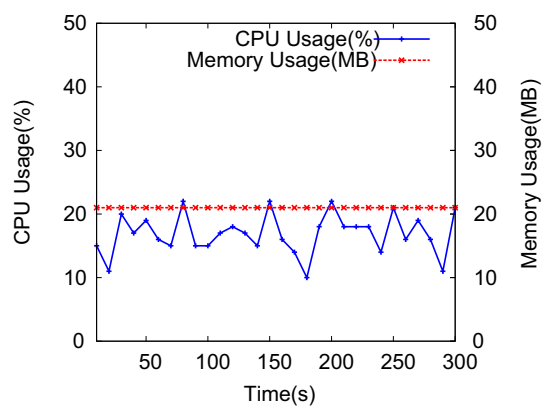

(b) Memory and CPU Usage

Fig. 6. Evaluation of Mobile Twitter on Device

We used an HTC Hero phone with Android 2.1 OS to send packets to another one through Bluetooth with different packet interval and size. We recorded the voltages respectively before and after sending packets for 30 minutes at different packet intervals. Figure 6(a) shows that the Bluetooth power consumption is relatively small. With a fixed packet interval, smaller packet size consumes less battery and with a fixed packet size, larger packet interval consumes less battery. We also used the PowerManager class in Android API to see that the application can last for about 14 hours when running exclusively all the time, i.e. doing both the packet communication and localization computation. Figure 6(b) shows that CPU usage is constantly between $10 \%$ to $22 \%$ and the memory usage is under $21 \mathrm{MB}$ over a typical 5 minute usage span, which are acceptable on real mobile devices.

\section{Discussion}

\subsection{Resolution Limitation}

As shown by Radhika Nagpal et al [11, there is a theoretical limitation for localization techniques in range-free category using only connectivity information. Although the ranging precision limitation of ad hoc sensing capabilities posed serious challenges to MobiAmorph, our problem settings is for users in urban environment, where user scale and pedestrian mobility can be used to our advantage. With altruistic users' sharing of its accurate GPS location, our localization algorithm has higher coverage and accuracy than previous ones.

\subsection{Privacy and Security for Adoption}

To increase the adoption of Mobile Twitter, we need to have security extensions to our localization technique and encourage GPS information sharing among users. To be a secured localization technique, MobiAmorph needs to handle malicious seeds that will inject fake location information, and relay nodes that may 
corrupt the hop count and replay packets. Authentications by digital signatures are necessary to sign up seed's messages. Key establishments mechanisms like random key predistribution [15] can establish keys using symmetric cryptography, which is suitable for MobiAmorph algorithm as bidirectional verification is possible in our case. For relay attack, we can use defense techniques like synchronized clocks [16] with additional hardware.

MobiAmorph has good properties for security extension. First it is based on limited hop count and connectivity information when a user is moving around. So an attacker's damage is very temporary, as long as the user can receive authentic information in the future. This is the mobility issue to our benefits. So MobiAmorph is similar to MCL, both of which are less susceptible to malicious seeds than other static approaches. We also need to develop meaningful mobile social applications that encourage the sharing of location information among mobile users. But application message should be encrypted for privacy protection, while anonymized localization should be abstracted as an independent service among mobile users.

\subsection{Pedestrian Mobility Model}

Since we are targeting the usage of Mobile Twitter for geosocial networking application by mobile pedestrians in urban environment, users may carry a smart phone walking around with independent or group interest down the road. Realistic mobility model like Urban Pedestrian Flows (UPF) [17] can be applied to see the effects of various ad hoc localization algorithms.

\section{Conclusion and Future Work}

We studied the problem of ad hoc localization with the help of GPS information suitable for urban environment with pedestrians. We proposed the MobiAmorph algorithm that can localize non-GPS mobile users with location information propagated from GPS-enabled mobile devices. We compared it with other two distributed range-free localization algorithms. MobiAmorph is shown to be superior with high accuracy and coverage under various settings. In general, increasing the seed ratio among the same number of nodes or shortening the packet interval of location forwarding will improve accuracy and coverage, but the tradeoff need to be understood to determine appropriate deployment parameters.

The Mobile Twitter application is developed with the MobiAmorph algorithm on the Android mobile device, using Bluetooth, GPS and Google Maps. The application performance is satisfactory in real life situations. By analysis of the performance tradeoff, we figured out the recommended settings to Mobile Twitter that can perform with low power consumed by localization computation and 
Bluetooth communication. Future works include evaluation of algorithms under irregular radio model, instead of perfect circle.

Acknowledgments. This research work is supported in part by HKU Seed Funding Program for Basic Research (Grant No. 10400018) and China 863 grant 2006AA01A111 (CNGrid). The authors thank Ip Chun Yin, Kwok Ka Ho, Kwok Wan Lai for their initial prototype implementation of this work.

\section{References}

1. Facebook, http://www.facebook.com

2. MySpace, http://www.myspace.com

3. Twitter, http://twitter.com

4. Geosocial networking, http://en.wikipedia.org/wiki/Geosocial_networking

5. GPS-Enabled, L.B.S.: Subscribers Will Total 315 Million in Five Years, http://www . abiresearch.com/abiprdisplay.jsp?pressid=731

6. Huang, R.: Probabilistic localization of mobile ad hoc networks. Phd Thesis, University of Texas Arlington (2006)

7. Savvides, A., Han, C.C., Strivastava, M.B.: Dynamic fine-grained localization in Ad-Hoc networks of sensors, pp. 166-179. ACM Press, New York (2001)

8. Niculescu, D., Nath, B.: Ad hoc positioning system (APS) using AOA. In: Twenty-Second Annual Joint Conference of the IEEE Computer and Communications(INFOCOM 2003), vol. 3, pp. 1734-1743. IEEE, Los Alamitos (2003)

9. Bahl, P., Padmanabhan, V.: RADAR: an in-building RF-based user location and tracking system. In: Nineteenth Annual Joint Conference of the IEEE Computer and Communications Societies (INFOCOM 2000), vol. 2, pp. 775-784. IEEE, Los Alamitos (2000)

10. Hu, L., Evans, D.: Localization for mobile sensor networks. In: 10th Annual international Conference on Mobile Computing and Networking, pp. 45-57. ACM, New York (2004)

11. Nagpal, R., Shrobe, H.E., Bachrach, J.: Organizing a Global Coordinate System from Local Information on an Ad Hoc Sensor Network. In: Zhao, F., Guibas, L.J. (eds.) IPSN 2003. LNCS, vol. 2634, pp. 333-348. Springer, Heidelberg (2003)

12. Niculescu, D., Nath, B.: DV Based Positioning in Ad Hoc Networks. Telecommunication Systems, 267-280 (2003)

13. Konishi, K., Maeda, K., Sato, K., Yamasaki, A., Yamaguchi, H., Higashino, T., Yasumoto, K.: MobiREAL Simulator Evaluating MANET Applications in Real Environments. In: 13th IEEE International Symposium on Modeling, Analysis, and Simulation of Computer and Telecommunication Systems, pp. 499-502. IEEE, Los Alamitos (2005)

14. Fujii, S., Uchiyama, A., Umedu, T., Yamaguchi, H., Higashino, T.: An Off-line Algorithm to Estimate Trajectories of Mobile Nodes Using Ad-hoc Communication (concise contribution). In: Sixth Annual IEEE International Conference on Pervasive Computing and Communications (PerCom 2008), pp. 117-124. IEEE, Los Alamitos (2008)

15. Eschenauer, L., Gligor, V.D.: A key-management scheme for distributed sensor networks. In: Conference on Computer and Communications Security, pp. 41-47. ACM, New York (2002) 
16. Hu, Y.C., Perrig, A., Johnson, D.: Packet leashes: a defense against wormhole attacks in wireless networks. In: Twenty-Second Annual Joint Conference of the IEEE Computer and Communications (INFOCOM 2003), pp. 1976-1986. IEEE Societies, Los Alamitos (2003)

17. Maeda, K., Sato, K., Konishi, K., Yamasaki, A., Uchiyama, A., Yamaguchi, H., Yasumoto, K., Higashino, T.: Getting urban pedestrian flow from simple observation: realistic mobility generation in wireless network simulation, Montreal, Quebec, Canada, pp. 151-158. ACM, New York (2005) 